\title{
The Impact of Routine Molecular Screening for SARS-CoV-2 in Patients Receiving Anticancer Therapy: An Interim Analysis of the Observational COICA Study
}

\author{
Giuseppe Di Lorenzo ${ }^{\mathrm{a}}$ b Mario lervolino $^{c}$ Ferdinando Primiano $^{\mathrm{d}}$ \\ Maurizio D'Ambrosio ${ }^{\text {e }}$ Concetta Ingenito ${ }^{a}$ Luciana Buonerba $^{a}$ \\ Giuseppina Busto $^{\text {a }}$ Claudia Ferrara $^{a}$ Annamaria Libroia ${ }^{a}$ Gianluca Ragone ${ }^{a}$ \\ Ferdinando De Falco ${ }^{\text {a }}$ Ferdinando Costabile $^{a}$ Pietro Fimiani ${ }^{a}$ \\ Francesco Ugliano $^{\text {b }}$ Chiara Ranieri $^{b}$ Emilio Leo $^{\text {a }}$ Giandomenico Roviello $^{f}$ \\ Luca Scafuri $^{\mathrm{a}}$ Germano Guerra $^{\mathrm{b}}$ Carlo Buonerba ${ }^{\mathrm{a}}$

\begin{abstract}
a Oncology Unit, "Andrea Tortora" Hospital, ASL Salerno, Pagani, Italy; ${ }^{b}$ Department of Medicine and Health Sciences
"Vincenzo Tiberio", University of Molise, Campobasso, Italy; 'General Directorate, ASL Salerno, Salerno, Italy;

${ }^{\mathrm{d} M e d i c a l}$ Directorate, ASL Salerno, Salerno, Italy; ${ }^{\mathrm{e} M e d i c a l}$ Directorate, DEA Nocera-Pagani-Scafati, ASL Salerno,

Nocera Inferiore, Italy; fDepartment of Health Sciences, University of Florence, Florence, Italy
\end{abstract}

\section{Keywords}

SARS-CoV-2 $\cdot$ Molecular screening $\cdot$ Cancer $\cdot$ COVID-19

\begin{abstract}
Introduction: Cancer aggravates COVID-19 prognosis. Nosocomial transmission of SARS-CoV-2 is particularly frequent in cancer patients, who need to attend hospitals regularly. Since March 2020, all cancer patients having access to the Oncology Unit at the "Andrea Tortora" Hospital (Pagani, Salerno - referred to as "the Hospital") as inpatients or outpatients receiving intravenous therapy have been screened for SARS-CoV-2 using RT-PCR nasal swab. The ongoing COICA (COVID-19 infection in cancer patients) study is an ambispective, multicenter, observational study designed to assess the prognosis of SARS-CoV-2 infection in cancer pa-
\end{abstract}

tients. The aim of the study presented here was to explore potential differences in COVID-19-related outcomes among screening-detected versus nonscreening-detected SARSCoV-2-infected patients. Methods: The COICA study enrolled cancer patients who had received any anticancer systemic therapy within 3 months since the day they tested positive for SARS-CoV-2 on RT-PCR. The target accrual is 128 patients, and the study was approved by the competent Ethics Committee. Only the subgroup of patients enrolled at the Hospital was considered in this unplanned interim analysis. Logistic regression analysis was used to evaluate the association of screening-based versus nonscreening-based diagnosis. Results: Since March 15, 2020, until August 15, 2021, a total of 931 outpatients and 230 inpatients were repeatedly screened for SARS-CoV-2 using RT-PCR nasal swab at the Hospital. Among these, 71 asymptomatic patients were pos- 
itive on routine screening and 5 patients were positive for SARS-CoV-2 outside the institutional screening. Seven patients died because of COVID-19. At univariate analysis, nonscreening- versus screening-detected SARS-CoV-2 infection was associated with significantly higher odds of $\mathrm{O}_{2}$ therapy $(\mathrm{OR}=16.2 ; 95 \% \mathrm{Cl}=2.2-117.1 ; p=0.006)$, hospital admission $(\mathrm{OR}=31.5 ; 95 \% \mathrm{Cl}=3.1-317.8 ; p=0.003)$, admission to ICU $(\mathrm{OR}=23.0 ; 95 \% \mathrm{Cl}=2.4-223.8 ; p=0.007)$, and death $(\mathrm{OR}=$ 8.8; $95 \% \mathrm{Cl}=1.2-65.5 ; p=0.034)$. Conclusion: Routine screening with RT-PCR may represent a feasible and effective strategy in reducing viral circulation and possibly COVID-19 mortality in patients with active cancer having repeated access to hospital facilities.

(c) 2021 S. Karger AG, Basel

\section{Introduction}

The COVID-19 pandemic has profoundly affected all aspects of healthcare, posing a multitude of challenges for patients, physicians, as well as public health policy makers [1]. In cancer patients, COVID-19 prevalence was reported to be higher compared to regional community prevalence [2]. Cancer patients are more likely to be infected with SARS-CoV-2 [3], to require intubation [4], and to die because of COVID-19 [3], which is likely the result of a complex interplay of multiple factors, including the effects of reduced organ and immune system function due to the underlying malignancy and anticancer medications [2]. Commonly accepted recommendations for COVID-19 management and prevention in cancer patients include reducing office visits, improving telemedicine services, preferring oral compared to intravenous medications, among others $[5,6]$. Nosocomial transmission of SARSCoV-2 is particularly frequent in cancer patients, who need to attend hospitals regularly for intravenous administration of anticancer therapies, office visits, management of uncontrolled symptoms, or adverse events due to therapy [7]. In this regard, according to the European Society for Medical Oncology, all cancer patients requiring hospital admission may be screened by using a RT-PCR pharyngeal swab to be performed within $48 \mathrm{~h}$ before admission [8]. Asymptomatic carriers of SARS-CoV-2 are likely to provide a major contribution to spreading the infection [9]. In this regard, the value of a molecular or serological screening in asymptomatic patients attending hospital facilities is likely to be critically dependent on the local epidemiological scenario, although no conclusive evidence obtained in prospective clinical trials supports its effects in terms of reduced COVID-19 mortality $[10,11]$.
In the Campania Region, large population-based screening campaigns have been conducted by assessing anti-SARS-CoV-2 antibodies in peripheral blood, as in the case of the locked down town of Ariano Irpino [12], while some facilities have used lateral flow chromatographic immunoassays to assess the presence of antiSARS-CoV-2 immunoglobulin $\mathrm{G}$ and immunoglobulin $\mathrm{M}$ antibodies in finger-stick whole-blood specimens for initial screening of asymptomatic cancer patients, followed by RT-PCR nasal swab in case of positivity [6]. The Oncology Unit at the "Andrea Tortora" Hospital (Pagani, Salerno referred to as "the Hospital" here) is the main Oncology Unit of Azienda Sanitaria Locale of Salerno (Campania Region, Italy), a public company that provides community-based health services to over 1 million citizens. Since March 2020, all cancer patients having access to the Hospital as inpatients or outpatients receiving intravenous therapy have been screened for SARS-CoV-2 as per the Hospital policy using RT-PCR nasal swab.

We here report an unplanned analysis involving patients recruited at the Hospital in the ongoing COICA (COVID-19 infection in cancer patients) study, an ambispective, multicenter, observational study designed to assess the prognosis of SARS-CoV-2 infection in cancer patients. In this work, we analyzed COVID-19 outcomes in cancer patients to explore potential differences among screening-detected versus nonscreening-detected SARSCoV-2-infected patients, with the intent to gather evidence regarding the potential utility of routine screening for SARS-CoV-2 in cancer patients.

\section{Materials and Methods}

The COICA study is an ongoing, multicenter, ambispective observational study including cancer patients who had received any anticancer systemic therapy within 3 months since the day they tested positive for SARS-CoV-2 on RT-PCR. COICA was mainly designed to capture the clinical course of SARS-CoV-2 infection in patients being treated for cancer, and its full results will be published once the accrual has been completed. Briefly, enrolled patients are required to sign an informed consent and are observed from the time of diagnosis of SARS-CoV-2 to confirmed recovery (defined as 2 negative RT-PCR tests in a raw performed at least 24 h apart) or death. RT-PCR must be performed following WHO guidelines [13]. The COICA study, with a target accrual of 128 patients, was approved by the competent Ethics Committee and conducted according to the principles of the Helsinki Declaration.

We here report a preliminary analysis of patients enrolled at the Hospital, updated as of October 1, 2021. The unplanned analysis presented here was conceived to assess potential differences in COVID-19 outcomes among screening-based versus nonscreening-based SARS-CoV-2 diagnosis. Institutional screening for SARS-CoV-2 was conducted for all cancer patients having access 
to the Hospital as either inpatients or outpatients receiving intravenous therapy, who had to be screened for SARS-CoV-2 using RT-PCR nasal swab the day before they were admitted to the Hospital. Screening was omitted if the patient was asymptomatic and had tested negative for SARS-CoV-2 on RT-PCR within the previous 20 days. Only patients enrolled at the Hospital, where $>90 \%$ of patients had been recruited in the COICA study, were considered in this analysis, in order to minimize potential sources of distortion associated with different institutional screening protocols. Screening-diagnosed patients were defined as those asymptomatic patients who were diagnosed with SARS-CoV-2 following the institutional screening program. Nonscreening-diagnosed patients were defined as those who had been diagnosed outside the institutional screening for any reason. Asymptomatic patients were defined as individuals without a recent history of cough or fever and with no more than a single symptom among diarrhea, joint pain, headache, vomiting, asthenia, sore throat, muscle pain, and loss of taste or smell, as others have done [12].

Descriptive statistics and frequency counts were used to summarize characteristics of the study population. Median numbers were presented with interquartile ranges (IQRs), unless specified otherwise. Logistic regression analysis was used to evaluate the association of screening-based versus nonscreening-based diagnosis along with other available variable of potential interest with SARS$\mathrm{CoV}$-2-related outcomes. All tests were 2 -sided, and a value of $p \leq$ 0.05 was considered statistically significant. All statistical analyses were conducted using R 3.5.2.

\section{Results}

Since March 15, 2020, until August 15, 2021, a total of 76 patients were enrolled in the COICA study at the Hospital and represent the study cohort assessed in this work. Of these, the majority (71\%) were enrolled in 2020. Among these, 71 asymptomatic patients were found to be positive on routine screening among a total of 931 outpatients and 230 inpatients who were repeatedly screened for SARS-CoV-2 using RT-PCR nasal swab at the "Andrea Tortora" Hospital in Pagani during the same time period. Only 5 patients were found to be positive for SARS-CoV-2 outside the institutional screening after being tested either because of symptomatic (4 cases) or because of incidental finding of interstitial pneumonia on CT scans. With the exception of 1 patient who was enrolled in August 2021, all patients were enrolled before April 2021. All patients were followed up until confirmed negative RT-PCR or death. None of the patients had been vaccinated against SARS-CoV-2, which is consistent with the fact that the vaccination campaign for cancer patients started in Campania in March 2021. Breast (19.74\%) and prostate cancer (19.74\%) were the 2 most frequent malignancies in the cohort. The cohort consisted of 47 males (61.84\%). Median age was 62 years (IQR,

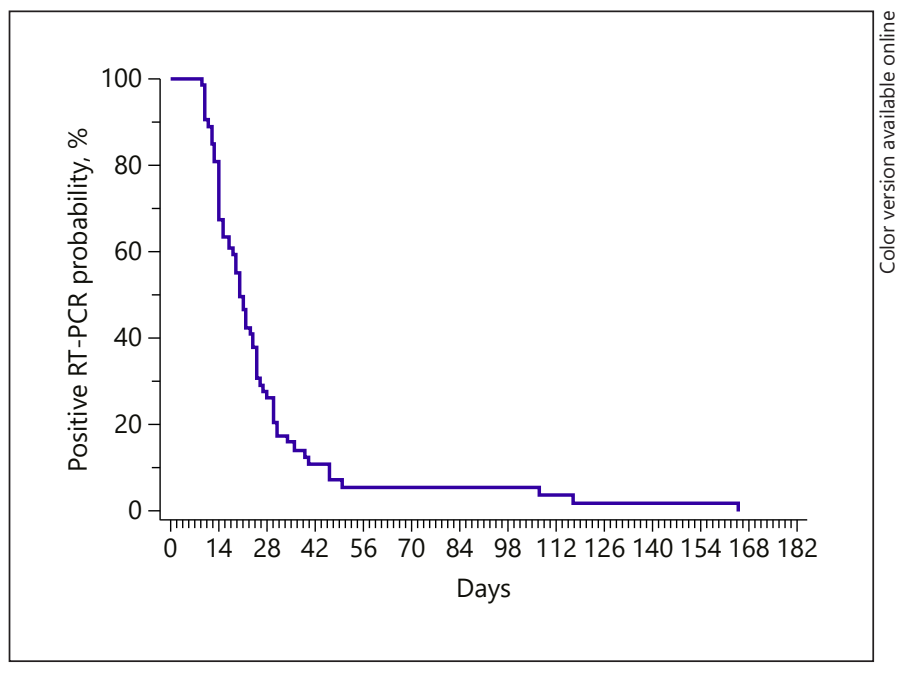

Fig. 1. Time to confirm negative RT-PCR.

55-72). Approximately half of the patients had received some antineoplastic systemic therapy within 30 days of diagnosis. Approximately 72 per cent of patients presented metastatic cancer, and about seventy-seven per cent presented some SARS-CoV-2-related symptoms during the observation. CT-confirmed lung pneumonitis was reported in 15 patients (19.74\%), while 12 (18.82\%) had to be admitted to the Hospital. O2 therapy was required in 9 patients (11.84\%), while 4 had to be admitted to the intensive care unit (ICU) (5.26\%). Seven patients died because of COVID-19 after a median of 19 days (IQR, 11-28). These 7 patients ( 2 males and 5 females) had been, respectively, diagnosed with ovarian (2 patients), head and neck (1 patient), kidney ( 1 patient), uterine ( 1 patient), breast ( 1 patient), and lung cancer ( 1 patient), and 6 of them had metastatic disease. Furthermore, 6 of them had to be admitted to the Hospital and 4 of them had to be admitted to the ICU. Antibiotics, heparin, and corticosteroids were administered to all of them (Table 1).

Median time to confirmed negative test was 20 days $(95 \% \mathrm{CI}=17-24)$, as shown in Figure 1. Any anti-COVID-19 treatment was administered in 49 (64.47\%) patients. Among these, antibiotics (67.3\%), heparin (30.6\%), and corticosteroids $(40.8 \%)$ were commonly administered.

At univariate analysis, nonscreening- versus screening-detected SARS-CoV-2 infection was associated with significantly higher odds of requiring $\mathrm{O} 2$ therapy $(\mathrm{OR}=$ $16.2 ; 95 \% \mathrm{CI}=2.2-117.21 ; p=0.006)$, hospital admission $(\mathrm{OR}=31.5 ; 95 \% \mathrm{CI}=3.1-317.8 ; p=0.003)$, admission to 
Table 1. Characteristics of the study population ( $n=76$ cases)

\begin{tabular}{|c|c|c|c|}
\hline \multicolumn{2}{|l|}{ Gender } & \multicolumn{2}{|l|}{ Symptomatic } \\
\hline Females & $29 / 76(38.16)$ & No & $17 / 76(22.37)$ \\
\hline \multicolumn{2}{|l|}{ Any antineoplastic treatment within 30 days } & \multicolumn{2}{|l|}{ Diagnosis } \\
\hline Yes & $37 / 76(48.68)$ & Screening & $71 / 76(93.42)$ \\
\hline Prostate cancer & $15 / 76(19.74)$ & Yes & $15 / 76(19.74)$ \\
\hline Ovarian cancer & $5 / 76(6.58)$ & No & $4 / 76(5.26)$ \\
\hline Merkel cell carcinoma & $1 / 76(1.32)$ & Admitted to the Hospital & \\
\hline Lung cancer & $7 / 76(9.21)$ & Yes & 12/76 (15.79) \\
\hline Breast cancer & $15 / 76(19.74)$ & No & $64 / 76(84.21)$ \\
\hline Colon cancer & $7 / 76(9.21)$ & Dead because of COVID-19 & \\
\hline Kaposi's sarcoma & $1 / 76(1.32)$ & No & $27 / 76(35.53)$ \\
\hline Pancreatic cancer & $1 / 76(1.32)$ & $\mathrm{O}_{2}$ therapy required & \\
\hline Testicular cancer & $3 / 76(3.95)$ & Yes & $9 / 76(11.84)$ \\
\hline Head and neck cancer (tongue cancer) & $1 / 76(1.32)$ & No & $67 / 76(88.16)$ \\
\hline Uterus cancer & $2 / 76(2.63)$ & ICU & \\
\hline Gastric cancer & $2 / 76(2.63)$ & Yes & $4 / 76(5.26)$ \\
\hline Urothelial carcinoma & $1 / 76(1.32)$ & No & $72 / 76(94.74)$ \\
\hline \multicolumn{2}{|c|}{ Antineoplastic agent(s) administered within 30 days } & \multicolumn{2}{|l|}{ Anti-COVID-19 treatment } \\
\hline Chemotherapy-based & & Antibiotics & \\
\hline Carboplatin-paclitaxel & $2 / 37(5.41)$ & Azithromycin & $21 / 49(42.86)$ \\
\hline FOLFOX-bevacizumab & $2 / 37(5.41)$ & Ceftriaxone & $2 / 49(4.08)$ \\
\hline Cisplatin-pemetrexed-pembrolizumab & $1 / 37(2.70)$ & Levofloxacin & $2 / 49(4.08)$ \\
\hline Paclitaxel & $1 / 37(2.70)$ & Prednisone & $10 / 49(20.41)$ \\
\hline Gemcitabine & $1 / 37(2.70)$ & Dexamethasone & $7 / 49(14.29)$ \\
\hline Paclitaxel-ramucirumab & $1 / 37(2.70)$ & Betamethasone & $3 / 49(6.12)$ \\
\hline Epirubicin-cyclophosphamide & $1 / 37(2.70)$ & FANS & \\
\hline Docetaxel-cyclophosphamide & $1 / 37(2.70)$ & Ibuprofen & $3 / 49(6.12)$ \\
\hline Hormonal therapy & & Ketoprofen & $1 / 49(2.04)$ \\
\hline Abiraterone & $3 / 37(8.11)$ & Heparin & \\
\hline Enzalutamide & $3 / 37(8.11)$ & Enoxaparin & $11 / 49(22.44)$ \\
\hline Immunotherapy-based & & Nadroparin & $3 / 49(6.12)$ \\
\hline Nivolumab & $2 / 37(5.41)$ & Parnaparin & $1 / 49(2.04)$ \\
\hline Atezolizumab & $1 / 37(2.70)$ & Other therapies & \\
\hline Pembrolizumab-axitinib & $1 / 37(2.70)$ & Lopinavir/ritonavir & $2 / 49(4.08)$ \\
\hline Targeted therapy & & Omeprazole & $1 / 49(2.04)$ \\
\hline Pertuzumab-trastuzumab & $4 / 37(10.81)$ & Tocilizumab & $1 / 49(2.04)$ \\
\hline Alectinib & $1 / 37(2.70)$ & Vitamin C & $1 / 49(2.04)$ \\
\hline Pazopanib & $1 / 37(2.70)$ & Acetylsalicylic acid & $1 / 49(2.04)$ \\
\hline Metastatic & & Paracetamol & $5 / 49(10.20)$ \\
\hline
\end{tabular}


Table 2. Univariate analysis of COVID-19-related outcomes

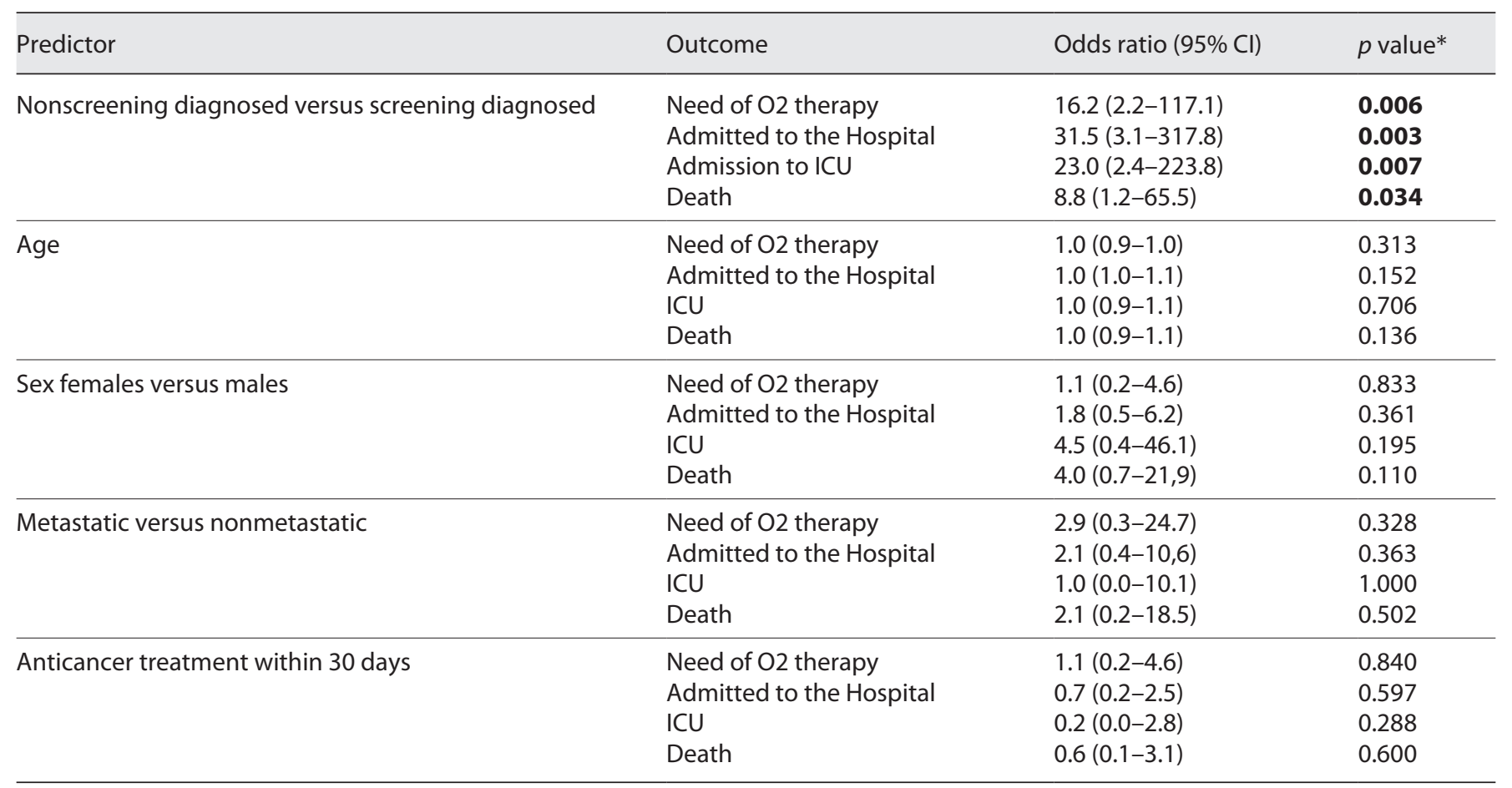

* Significant $p$ values are highlighted in bold.

ICU $(\mathrm{OR}=23.0 ; 95 \% \mathrm{CI}=2.4-223.8 ; p=0.007)$, and death $(\mathrm{OR}=8.8 ; 95 \% \mathrm{CI}=1.2-65.5 ; p=0.034)$. None of the other potential predictors explored were significant (Table 2).

\section{Discussion}

The main objective of the COICA study was to add evidence to the existing body of the literature suggesting that COVID-19 prognosis is negatively affected by a previous or concomitant cancer diagnosis. In fact, in 1 retrospective analysis of 557 consecutive COVID-19 patients, of whom 46 had active cancer, an overall fatality rate of 50\% (95\% CI: $34.9-65.1$ ) versus 20.2\% (95\% CI: $16.8-$ 23.9 ) in patients with versus without cancer was reported, with a median OS of 14 versus 35 days, respectively [14]. Consistent results have been obtained by other researchers $[15,16]$. Finally, in a retrospective case-control study conducted by analyzing medical records of 2,523,920 cancer patients, patients with cancer and COVID-19 had significantly worse outcomes (hospitalization rate, $47.46 \%$; death rate, $14.93 \%$ ) compared to nononcological patients with COVID-19 (hospitalization rate, $24.26 \%$; death rate, $5.26 \%)(p<0.001)$ [17].

Based on these findings, the optimal strategy for early detection of SARS-CoV-2 in patients who periodically have access to hospital facilities to receive anticancer therapy remains to be established and contextualized in the evolving epidemiologic scenario. In this work, we presented clinical outcomes related to SARS-CoV-2 infection in patients receiving systemic antineoplastic treatment at the coordinating center of the COICA study and reported a $9.21 \%$ death rate, with $11.84 \%$ of patients requiring $\mathrm{O}_{2}$ therapy, $15.79 \%$ requiring to be admitted to the Hospital, and $5.26 \%$ being admitted to the ICU. These findings appear to be more favorable compared to those mentioned above [14-17]. When we explored potential predictors of COVID-19-related outcomes, we found that nonscreening- versus screening-detected SARSCoV-2 infection was associated with significantly higher odds of death, needing $\mathrm{O}_{2}$ therapy, being admitted to hospital as well as being admitted to ICU. Although definitive conclusions can be drawn only in the context of a randomized interventional trial, our data seem to support the benefits of routine screening for SARS-CoV-2. In fact, the 
institutional screening policy adopted at the Hospital allowed to identify 71 asymptomatic cases overall, that is, approximately $6 \%$ of the 1,161 screened patients in the period considered. Our positive test rate was higher compared to other experiences. In fact, in a retrospective study including 1,226 cancer patients who were offered to be screened for SARS-CoV-2, a positive test was identified only in 10 patients (approximately $1 \%$ ), with only 7 patients being asymptomatic at the time of testing, which translated into an asymptomatic infection prevalence of 0.6\% (95\% CI [0.15-0.99] [2]. Similarly, in another study conducted in 2,691 cancer patients who underwent asymptomatic screening, only $1.6 \%$ of patients were SARSCoV-2 positive, with $11.6 \%$ of the cohort developing COVID-19-related symptoms during the course of the disease [18]. Differently from these findings, we reported that in our cohort of initially 71 asymptomatic patients, $76 \%$ developed symptoms over the course of the disease, which suggests that our approach may have represented a truly effective strategy leading to early SARS-CoV-2 diagnosis. One possible interpretation of this unexpected finding is that patients might have been more frequently exposed to the virus just before they were screened, considering that patients on active therapy at the Hospital generally preferred to take a blood draw in any external laboratory next to their home a few days before and were screened for SARS-CoV-2 the day before they were scheduled for therapy at the Hospital.

We recognize that our study presents multiple limitations. First of all, it is an unplanned analysis of an observational study, which therefore does not allow to draw any conclusion about the efficacy of the screening strategy adopted, and it can only provide hypothesis-generating results. Second, the COICA trial was not designed to include all patients diagnosed at any participating center, which may have been responsible for a selection bias. In fact, only 5 cancer patients were diagnosed outside the institutional screening. To the best of the investigators' knowledge, all patients with a SARS-CoV-2 diagnosis at the Hospital were offered to participate to the COICA trial and were ultimately included, although we are unaware of how many SARS-CoV-2 cases among cancer patients treated at the Hospital were missed. Third, the data presented were collected in a pre-vaccination scenario, with none of the enrolled patients being vaccinated, so they cannot be extrapolated in the current epidemiologic scenario, with the majority of patients being vaccinated. Fourth, the sample size of 76 patients, with only 5 patients diagnosed outside the institutional screening, is limited. Nevertheless, this analysis presented here has the strength to reflect the results obtained as a single center where a single screening protocol was followed, with all enrolled patients followed up until recovery or death.

In conclusion, this unplanned analysis of the COICA trial performed at a single center reported an unexpected high rate of screening-detected SARS-CoV-2 infections in cancer patients. The potential implications for COVID-19-associated outcomes are unknown, and the generalizability of our results is limited. Routine screening with RT-PCR may represent a feasible and effective strategy in reducing viral circulation and possibly COVID-19 mortality in patients with active cancer having repeated access to hospital facilities.

\section{Statement of Ethics}

Written informed consent was obtained from participants (or their parent/legal guardian/next of kin) to participate in the study. This study protocol was reviewed and approved by the local Ethics Committee Campania Sud, approval No. 2020-3JF. The study was conducted according to the criteria set by the Declaration of Helsinki.

\section{Conflict of Interest Statement}

Dr. Di Lorenzo serves as an editorial board member of Oncology. All the other authors have no conflicts of interest to declare.

\section{Funding Sources}

No funding was obtained for this work.

\section{Author Contributions}

Giuseppe Di Lorenzo, Luciana Buonerba, Concetta Ingenito, and Carlo Buonerba performed study concept and design. All authors involved in acquisition of data. Giuseppe Di Lorenzo and Carlo Buonerba performed analysis of data. All authors involved in interpretation of data. Giuseppe Di Lorenzo and Carlo Buonerba drafted the manuscript. All authors performed critical revision of the manuscript for important intellectual content.

\section{Data Availability Statement}

Anonymized data are available upon request to the corresponding author without any restrictions. 


\section{References}

1 Tuech JJ, Gangloff A, Di Fiore F, Benyoucef A, Michel P, Schwarz L. The day after tomorrow: how should we address Health System Organization to treat cancer patients after the peak of the COVID-19 epidemic? Oncology. 2020;98:827-35.

2 Lee LYW, Hill T, Topping O, Tilby M, Baker M, Greig J, et al. Utility of COVID-19 screening in cancer patients. Cancer Cell. 2020;38: 306-7.

3 Roel E, Pistillo A, Recalde M, Fernández-Bertolín S, Aragón M, Soerjomataram I, et al. Cancer and the risk of COVID-19 diagnosis, hospitalisation, and death: a populationbased multi-state cohort study including $4,618,377$ adults in Catalonia, Spain. Int J cancer. 2021.

4 Miyashita H, Mikami T, Chopra N, Yamada T, Chernyavsky S, Rizk D, et al. Do patients with cancer have a poorer prognosis of $\mathrm{CO}$ VID-19? An experience in New York City. Ann Oncol. 2020;31:1088-9.

5 Ingenito C, Buonerba L, Ferrara C, Busto G, Libroia A, Ragone G, et al. Coronavirus disease 2019 emergency and cancer in the South of Italy: what's new for the oncologist? Front Med. 2020;7:189.

6 Di Lorenzo G, Buonerba L, Ingenito C, Crocetto F, Buonerba C, Libroia A, et al. Clinical characteristics of metastatic prostate cancer patients infected with COVID-19 in South Italy. Oncology. 2020;98(10):743-7.
7 Du Q, Zhang D, Hu W, Li X, Xia Q, Wen T, et al. Nosocomial infection of COVID-19: a new challenge for healthcare professionals (Review). Int J Mol Med. 2021;47:31.

8 Curigliano G, Banerjee S, Cervantes A, Garassino MC, Garrido P, Girard N, et al. Managing cancer patients during the COVID-19 pandemic: an ESMO multidisciplinary expert consensus. Ann Oncol. 2020;31:1320-35.

9 Di Lorenzo G, Di Trolio R. Coronavirus disease (COVID-19) in Italy: analysis of risk factors and proposed remedial measures. Front Med. 2020;7:140.

10 Sun R, Achkar S, Ammari S, Bockel S, Gallois E, Bayle A, et al. Systematic screening of COVID-19 disease based on chest CT and RTPCR for cancer patients undergoing radiation therapy in a coronavirus french hotspot. Int J Radiat Oncol Biol Phys. 2021;110(4):947-56.

11 Xie Z, Saliba AN, Abeykoon J, Majeed U, Almquist DR, Wiedmeier-Nutor JE, et al. Outcomes of COVID-19 in patients with cancer: a closer look at pre-emptive routine screening strategies. JCO Oncol Pract. 2021; 17(9):e1382-93.

12 Cerino P, Coppola A, Volzone P, Pizzolante A, Pierri B, Atripaldi L, et al. Seroprevalence of SARS-CoV-2-specific antibodies in the town of Ariano Irpino (Avellino, Campania, Italy): a population-based study. Future Science OA. 2021;7(4):FSO673.
13 Corman V, Tobias B, Brünink S, CD, Koopmans M, Zambon M. Diagnostic detection of 2019-nCoV by real-time. RT-PCR; 2020.

14 Bertuzzi AF, Ciccarelli M, Marrari A, Gennaro N, Dipasquale A, Giordano L, et al. Impact of active cancer on COVID-19 survival: a matched-analysis on 557 consecutive patients at an Academic Hospital in Lombardy, Italy. Br J Cancer. 2021;125(3):358-65.

15 Nader Marta G, Colombo Bonadio R, Nicole Encinas Sejas O, Watarai G, Mathias Machado MC, Teixeira Frasson L, et al. Outcomes and prognostic factors in a large cohort of hospitalized cancer patients with COVID-19. JCO Glob Oncol. 2021;7:1084-92.

16 Martin S, Kaeuffer C, Leyendecker P, Tuzin $\mathrm{N}$, Tazi Y, Schaff-Wendling F, et al. COVID-19 in patients with cancer: a Retrospective Study of 212 cases from a French SARS$\mathrm{CoV}-2$ cluster during the first wave of the COVID-19 pandemic. Oncologist. 2021;26(9): e1656-9.

17 Wang Q, Berger NA, Xu R. Analyses of risk, racial disparity, and outcomes among US patients with cancer and COVID-19 infection. JAMA Oncol. 2021;7(2):220-7.

18 Shaya J, Cabal A, Nonato T, Torriani F, Califano J, Lippman S, et al. Asymptomatic detection of SARS-CoV-2 among cancer patients receiving infusional anti-cancer therapy. Cancer Med. 2021. 